\title{
DYNAMIC VIRTUAL ORGANIZATIONS, OR NOT SO DYNAMIC?
}

\author{
L. M. Camarinha-Matos', H. Afsarmanesh' \\ ${ }^{\prime}$ New University of Lisbon, Faculty of Sciences and Technology \\ Quinta da Torre, 2829-516 Monte Caparica, PORTUGAL, cam@uninova.pt \\ ${ }^{2}$ University of Amsterdam, Faculty of Science \\ Kruislaan 403, 1098 SJ Amsterdam, THE NETHERLANDS, hamideh@science.uva.nl
}

\begin{abstract}
Expectations created by the early rapid developments in virtual organizations, hoping to resolve the main challenges in the area and continuous emergence of new related facilities are still far from full materialization. Being a multidisciplinary area that requires the participation of experts in ICT, theory of organizations, sociology, economy, law, etc., there is primarily a need for further developments in terms of modeling and better understanding of concepts, as well as supporting infrastructures and tools. Harmonization of the current approaches and establishment of common reference models and practices is also a pre-requisite. In this context a brief characterization of the state of the art is made and several open research issues are introduced.
\end{abstract}

\section{INTRODUCTION}

\subsection{Collaborative Organizations}

Collaborative and networked organizations have gained momentum during the last years changing the way that commercial, industrial, cultural and social activities are organized. In addition to the rapid evolution of traditional supply chains and outsourcing environments, a growing trend nowadays consists of tasks performed by autonomous teams set up as independent contractors and linked by a network. These teams usually come together, some times in temporary combinations, to tackle various projects, and may dissolve once the work is done. Consider the wide variety of recent radical outsourcing in design for manufacturing, printing industry, software development, or the film industry: in almost every case, in one way or another "Smart Organizations", "Virtual Organizations" or "Networked Organizations" represent a collaborative configuration.

The proliferation and variety of terms applied to forthcoming business structures, such as blurring borders in organizations, alliances, partnerships, aggregations, value constellations, business webs, and business ecosystems, show only the surface of a fast changing paradigm. During the last decade, and in parallel with the development and spreading of Internet technologies, traditional collaboration networks have found new leveraging tools and new collaborative business forms have emerged. New technologies, business processes, and organizational life forms supported by networking tools "invade" all traditional businesses and organizations what requires thinking in terms of whole systems, i.e. seeing each business as part of a wider economic ecosystem and environment. The new collaborative paradigm needs to be better understood and properly supported, not only in terms of the needed 
technologies, but also through the development of adequate skills, tools \& mechanisms, culture and regulations. Complementarily, there is the emergence of new value systems for provided products and services that need to be understood, embedded in the new business practices and supported by new tools.

In the current market scenario, recent trends in industry are also emphasizing the relevance of agility, understood as the ability to recognize and rapidly react and cope with the unpredictable changes in the environment [8], with a smooth adaptation of its entire structure to the new / current reality. The idea of highly dynamic organizations, that form themselves and acquire shape according to the needs and opportunities of the market and remain operational as long as these opportunities persist, suggests a number of benefits, among which the following can be emphasized:

- Agility: being the capability to recognize, rapidly react and cope with the unpredictable changes in the environment in order to achieve better responses to opportunities, shorter time-to-market, and higher quality with less investment.

- Complementary roles: enterprises seek for complementarities (creation of synergies) that allow them to participate in competitive business opportunities and new markets.

- Achieving dimension: especially in the case of SMEs, being in partnerships with others allow them to achieve critical mass and appear in the market with a larger "visible" size.

- Competitiveness: achieving the cost effectiveness, by proper division of subtasks among cooperating organizations.

- Resource optimization: smaller organizations sharing infrastructures, knowledge, and business risks.

- Innovation: being in a network opens the opportunities for the exchange and confrontation of ideas, the basis for innovation.

Considerable investments have been made, namely in Europe and the USA, in a large number of research projects applying new organizational forms, which have produced an abundant variety of specific solutions and broad awareness for the necessary organizational changes. Particularly in the case of Europe, given both its cultural background in business and the current emerging development investments, Europe is placed in a key leading position for the development of the organizational forms fit for the digital age. However, the research in many of these cases is highly fragmented being each project focused on solving specific problems and applying IT to partially design and develop a minimal B2B interaction mechanism to support its basic needs. As such, there is no effective consolidation/harmonization among them in order to have an effective impact. How to integrate all these fragmented research contributions and how to empower enterprises in both their regional and global competitiveness are open questions.

On the other hand proliferation of fast changing short-life non-interoperable technologies is a major obstacle for SMEs entering collaborative partnerships. The crash of many unrealistic "dot-com" ventures also slowed down some investments by the industry, but the well-founded research towards the development of adequate support infrastructures based on realistic business models has seen a continuous progress. In fact, while the basic networking infrastructures and the virtual organization (VO) / virtual enterprise (VE) paradigm are spreading, new forms of collaborative virtual communities / virtual communities of practice (VCP), i.e. stronger human-oriented organizations, are emerging. However, the wide variety of 
terms and concepts, most overlapping and without precise definitions, show the fundamental need to establish a clear baseline. In order to be efficient and competitive in their operation, the VOs and other collaborative organizations of the future have to rely on solid bases and strong methodological approaches.

\subsection{Technology Trends}

The availability of a large number of contributing elements (technologies, paradigms, best practices and models) constitutes a set of enabling factors for the opportunity of materializing concepts that although not new, were waiting for the enabling factors. Examples are:

a On VE / VO: Significant developments in support infrastructures, various experiments in regional clustering / networking, and various pilot cases of IT supported clusters.

口 On spreading, widening, and acceptance of the concept of "sharing": Computational resource sharing through GRID, collaborative engineering, Virtual Laboratories (VL), P2P-ERP/PDM Systems for dynamic SMEs, contracts and negotiation models (acquaintance models, auctions, etc.).

- On new organizational design, including structure and processes, and interactions with the environment.

ㅁ On the information and communication technology research advances: Web technology, standards and tools, communication security, federated information management, coordination theory and systems, component-based system design and developments, MAS technology and software mobility, GRID technology, mobile computing.

- Advances in knowledge representation, with particular focus on shared ontologies and imprecise knowledge representation / soft-computing.

- Basic technologies and tools for "traditional" Virtual Communities, still limited and not considering communities of practice under social contracts.

However, most of these technologies and concepts are in their infancy and under development, mostly fragmented and non-interoperable, requiring considerable effort to implement and configure comprehensive VO support infrastructures and operational methods. Even the most advanced infrastructures coming out of leading R\&D projects require complex configuration and customization processes hardly manageable by SMEs. There is a lack of common reference models, interoperable infrastructures, and general business support functionalities that constitute major obstacles to resources and information sharing.

\subsection{The Way Ahead}

There is a growing awareness that the VO developments should be based on contributions of multidisciplinary nature, namely from the Information and Communication Technologies, socio-economic, cognitive aspects, operations research, organizational, business management, legal, social security, and ethical areas. Some trends in this direction are:

․ New behavioral forms, which include new ways of work and even new moral and ethical attitudes;

- New cooperation agreements and social contracts;

New liability agreements and risk negotiation practices; 
- New ways of generating value for common developments;

- Correspondingly, new challenges on IPR and ownership identification; and

$\square$ Definition of legal frameworks affecting /addressing VOs.

Furthermore there is a need for mechanisms to empower human relationships as a way to induce creativity, to strengthen cohesion and sustainability and to reach responsiveness to market turbulence. However the developments in this area are still mostly technology-driven and achieved independently of each other and at different projects, thus repeatedly providing only very primitive interaction infrastructure among the organizations involved in the VO.

Understanding the emerging behavior and trends (how and why) is a challenge, requiring further developments in modeling, support mechanisms and tools, coordination principles and leadership, and establishment of collaboration support (virtual) institutions (e.g. e-notary, e-dispute-solving).

Similarly there is a need for planning and establishing new integrated metrics. Dynamic evaluation of the fractional value of processes (e.g. value features, rewarding principles, creation of enterprise-wide appreciation of new value features, trust arbitrators, auditability), need to be part of a comprehensive research and development initiative.

This paper summarizes some ideas under discussion in the framework of two international initiatives: IST THINKcreative and IFIP COVE. THINKcreative is an EC funded network of experts that aims at identifying and characterizing emerging organizational collaborative forms and their required infrastructures, modeling and application tools, and socio-organizational needs for the next 5, 10 and 20 years. COVE started as a project aiming to contribute to the harmonization and knowledge dissemination of world-wide research results on virtual organizations, and to foster needed collaborative developments. More recently COVE evolved to a permanent Working Group of the IFIP Technical Committee 5, the WG5.5.

Following sections discuss major aspects in VO: infrastructures, cluster management and VO creation, VO operation, evolution and dissolution, virtual laboratories and virtual communities. For each of these topics, a brief overview of the state of the art and major trends is presented, followed by a list of suggested key topics for further research.

\section{INFRASTRUCTURES FOR VIRTUAL ORGANIZATIONS}

Although the potential advantages of the Virtual Organizations are well known at the conceptual level [3], [7], their practical implantation is still far from the expectations. In order to leverage the potential benefits of the agile VE/VO paradigm, there is a need for flexible and generic infrastructures to support the full life cycle of the VO/VE, i.e. creation, operation, evolution and dissolution. Achieving such infrastructures is still a major challenge. The lack of a common and widely accepted reference model and infrastructure is still forcing every vertical development project to design and implement its own mini-infrastructures, deviating some resources from its main focus, while generating something only applicable to that project.

In fact, even the most advanced infrastructures coming out of leading R\&D projects still require complex configuration and customization processes, which are hardly manageable by SMEs. Infrastructures combining heterogeneous components 
from different vendors are also potentially unstable being difficult to determine which component (or tool provider) is responsible when something goes wrong with such complex systems. In spite of the fast growing technological developments, lack of proper interoperability mechanisms among enterprise applications is a major obstacle to agile VO/VEs. As complex organizations, formed by heterogeneous and autonomous entities, VOs need to deal with a combination of different technologies and a variety of application sources.

Although it is not a new subject but rather the central question in systems integration, interoperability remains as a critical topic in the agenda of VO/VE supporting infrastructures. In fact, effective cooperation in a VO/VE requires interoperation among the enterprise applications at two levels: (i) Intra-enterprise interoperability - comprising systems integration at the level of enterprise applications; and (ii) Inter-enterprise interoperability - comprising systems integration at the level of virtual organization (requiring that similar reference models are adopted). Furthermore, when discussing the interoperability approaches we need to carefully address the "life cycle" aspects as different enterprise technologies have quite different life cycles (e.g. ICT, manufacturing technology, products, services) and the components to be integrated may be at different stages of their life cycles.

Some of the main trends in the attempt to develop generic VO infrastructures include:

- Layer-based frameworks, which add a cooperation layer to the existing ICT platforms of the enterprises. Inter-enterprise cooperation is then performed via the interaction through these layers. Examples of this approach are early efforts in VE infrastructures, as represented by the NIIIP [11], PRODNET [3], [7], or VEGA [18] projects, that aimed at designing open platforms to support the basic information exchange and coordination needs in industrial virtual enterprises.

- Agent-based frameworks, including those approaches that represent enterprises as agents and the inter-enterprise cooperation as interactions in a distributed multi-agent system. Although less-developed than the layer-based approaches, various examples focusing the creation and operation of VOs can be found:

- Agents in VE creation - A growing number of works are being published on the application of multi-agent systems and market-oriented negotiation mechanisms for the VE formation [5], [10], [14].

- Agents in VE operation - Various projects have been addressing the dynamic scheduling and execution of distributed business processes [2], [12].

- Service-federation / service-market frameworks - According to this model, enterprises should be able to plug/unplug their services to/from service directories [4]. By means of proper "standard" service interface, the interoperability with other (requesting) enterprises, regardless of the heterogeneity associated with the actual implementation of the services themselves, is supported. This means that no matter how the service is actually implemented, in terms of the computer platform, operating system, programming language, internal modules, etc., there is a client service interface that can be managed by a lookup service and made available to other enterprises that may request it. The underlying concepts of the JINI architecture [15] show how to support, in a transparent way, a federation of service functions offered by different service suppliers and running on different nodes of a network. Although 
JINI may contribute, in theory, to facilitate the interoperability among services offered by different enterprises, its use in VO environments requires its extension to properly work in wide area networks and the development of additional support functionalities. For instance, the matter of access rights and enterprises information visibility is a very important issue, and therefore when searching and accessing a specific service type it is also relevant to determine both the supplier of the service and the requesting client, in order to check such visibility rights. It is also necessary to define the rules for both service specification/definition and service registration through the service interface. Furthermore, the general acceptance of the service interface by the service providers for developing services compliant with such rules is of great importance. Latest developments such as WSDL / SOAP represent a positive step in this direction.

Suggested research topics. Setting up an infrastructure for VO/VE still requires a large engineering effort, which represents a major obstacle for the implantation of this new organizational paradigm. Furthermore, it shall be noted that the fast evolution of the information technologies often presents a disturbing factor for nonIT companies. Therefore, further research effort is necessary towards the establishment of generic and interoperable infrastructures. Some open research issues include:

a Advanced federated information management, supporting the authorized information exchange, information integration mechanism, and collaborative work among organizations.

- Consideration of new assumptions in interoperability: (i) Interoperability is a continuous process as new technologies are emerging every day! (ii) Interoperability should become a "design principle" in software systems. (iii) Diversity / heterogeneity / autonomy of components must be accepted (not avoided). (iv) Co-existence of components with different life cycles and in different stages of their life cycles must be assumed.

- Progress towards an invisible, pervasive, and safe support infrastructure.

- Inter-domain transactions and recovery mechanisms.

$\square$ Effective integration of legacy systems.

- Tracking and auditing support services. Also self-identification (of products), allowing traceability and closing the gap between the physical world and the logical world.

\section{CLUSTER MANAGEMENT AND VO CREATION}

Although not well understood earlier, it is now clear that the formation of dynamic VO/VE requires an appropriate "breeding" or "nesting" environment (e.g. regional industry cluster) in order to guarantee basic requirements such as:

- Trust building ("trusting your partner" is a long-term process),

- Common infrastructures and agreed upon business practices (requiring substantial engineering / re-engineering effort),

- A sense of community and some sense of stability.

Industry clusters do not correspond to a new concept as a large number of related initiatives have emerged during the last decades, namely in Europe and the USA. But the advances in information and communication technologies now bring new 
opportunities to leverage the potential of this concept, namely by providing the adequate environment for the rapid formation of agile virtual organizations.

It is important to notice that there is a potential conflict between fast innovation and the stability requirements that are both part of the human nature. It is necessary to find harmonic solutions to conciliate the dynamism of the new business ecosystems and the needs for stability of individuals (and their families).

If long-term collaborative environments or business ecosystems are in place, it is viable to establish dynamic VO/VE as a rapid and optimized response to business opportunities and threats.

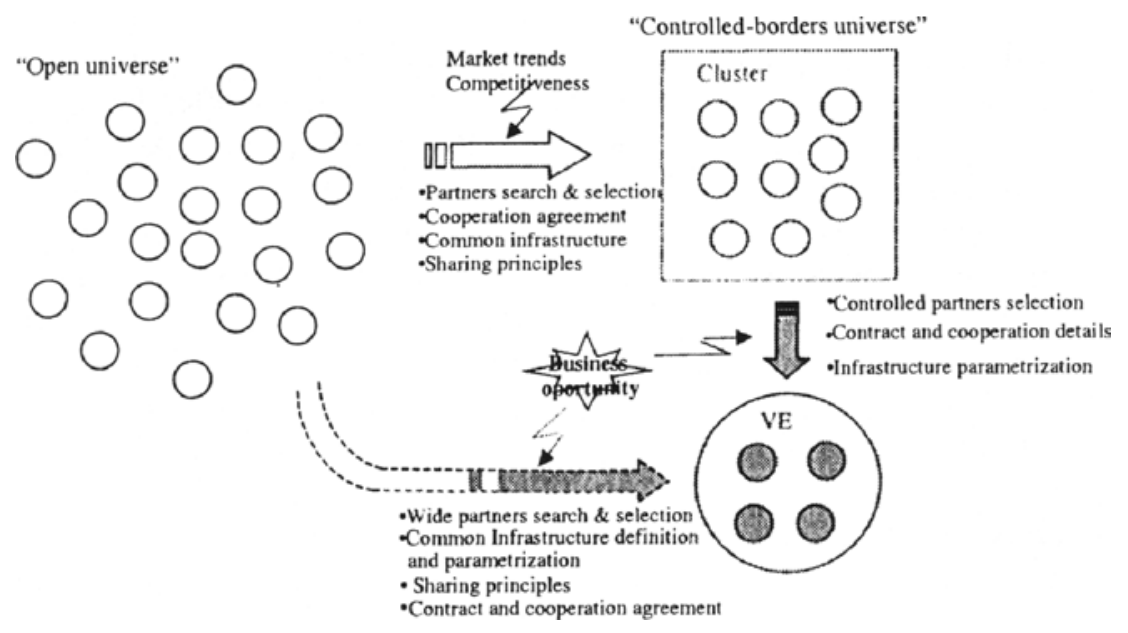

Figure 1 - Two approaches for VE formation

The Brazilian VIRTEC the ALFA COSME-VE, COWORK, and MASSYVE [13] are examples of early initiatives that have addressed specific aspects of creation and management of clusters of enterprises. Some of the aspects that have been subject of more attention are: Brokerage, partners search and selection (e-procurement), definition of cooperation rules (negotiation and contracting), etc.

One of the problems in partners search for VO creation is the availability of directories of companies / organizations where their profile (skills, resources, performance history, etc.) are represented in a standard format. The unavailability of a standard to represent these profiles has been a major obstacle. Recent developments (UDDI, WSDL, SOAP) represent promising steps in this direction. Nevertheless if the catalog is confined to a cluster it is easier to reach a common representation of profiles.

Some open research topics include:

- Management and configuration of local/regional clusters operating on specific vertical markets with specific business processes requirements.

- The full VO/VE creation framework.

D E-notary and certification services for networked organizations with special services such as: black lists, past performance, credentials, and best practices.

a Modeling and management of cooperation contracts and agreements.

a Methodologies for transforming existing organizations into VO-ready organizations. 


\section{VO OPERATION}

Coordination of distributed business processes (DBP) and activities is an important element in the VO operation. Most of the early approaches took a workflow-based approach, starting with the WfMC reference architecture [17] and experimenting with extensions for supervision of distributed processes (cross-organizational workflow) including some preliminary but very limited works on exception handling, multi-level coordination and its relationship to coordination roles of the VO members, and flexible workflow models to support less structured processes.

In terms of DBP planning and modeling some graphical languages have been suggested but a standard is still necessary in order to allow effective distribution / sharing of business processes. Proposals like PIF and PSL have been discussed but there is still more work to do. A recent European initiative, the UEML network, might contribute to some harmonization in the modeling area.

In terms of business support functions, most of the projects have addressed application specific cases. ERP vendors have been extending their monolithic single-enterprise-centric systems in order to comply with more dynamic supply chains and networks, but this is an area requiring more investment on generic functionalities.

Some open research topics:

- Coordination, administration and management of highly distributed activities, tasks, processes, and roles. Planning and supervision of distributed processes (including complex services).

Risk management / assessment tools.

- Design, development, provision and management of value-added services provided in the context of VE/VOs.

- Dynamic evaluation of revenues, rights and liabilities of every VE relationships, and dynamic selection of new partners in order to make the VE network more effective.

- Clarify / re-define notions of value in a society of relationships, focusing on the added-value contribution (how to measure it?) of each node and how to reward it in order to preserve the business ecosystem (dynamic sustainability concept). What is valuable, where does the value reside, who generates value? Cooperation adds an overhead that has to be compensated by the added-value and agility.

- Soft-modeling and reasoning with special decision support mechanisms for supply chain management, selection of partners or efficiency of VE relationships.

- E-contract management and adaptation to the legal frameworks.

- Advanced simulation models and tools for networked collaborative organizations.

- New business support functions and better integration with enterprise applications.

- New user interfaces, seeking an entertainment facet as a way to overcome cultural barriers. 


\section{VO EVOLUTION AND DISSOLUTION}

During the life cycle of a VO it is natural that some partner leaves the consortium and be replaced by a new organization. The termination of this collaboration process or even the ending of a VO, are subjects not properly addressed yet.

The consequences of the operation of a VO/VE cannot be simply discarded when the VO/VE dissolves. Most of these consequences are of a legal nature and shall be regulated by the cooperation agreements. That is the case, for instance, of the responsibility of customer support / product maintenance during the life cycle of the product / service generated by a VO/VE. Environment regulations are also forcing companies to plan provisions regarding the product disposal and recycling after its end of life.

Recent regulations in some countries also state that the liabilities regarding each component of a product may ultimately lie with the component's supplier. In the case of a network chain type of manufacturing this forces each node in the chain to keep track of the history of each component/sub-product that "passed by" this node. This is a functionality that is properly supported by most of the more advanced ERP systems, but not by most of the scheduling systems, for instance.

There are, however, several other less "material" issues which are more difficult to handle. One of these issues is the Intellectual Property Rights (IPRs) policy, namely for the post-dissolution phase and its consequences in terms of information accesses by the VE members. In some cases there is also the possibility that the VE evolves into a more permanent organization, a joint venture enterprise created by the VE members, to exploit the intellectual and industrial property results developed in cooperation. There is also considerable knowledge that can be elicited from the ending cooperation experience, namely the knowledge about what went right, what went wrong, partners performance / reliability, jointly defined business process templates, etc. Defining the ownership and access rights to this knowledge is not an easy task and requires further investigation.

Therefore, some important research topics include:

Definition of a legal and organizational framework for the termination of a cooperation process.

- Creation of mechanisms for handling post-cooperation IPRs and liabilities.

a Management functionalities to support the post-VO access to common resources and information.

Mechanisms to support extensive traceability.

\section{VIRTUAL LABORATORIES}

A Virtual Laboratory (VL) is another form of collaborative network, representing a heterogeneous, distributed problem solving environment that enables a group of researchers located in different geographical places to work together, sharing resources (equipments, tools, experimental data, etc.), i.e. a specialized form of VO.

In some domains requiring expensive equipment and a large critical mass of researchers, e.g. bio-informatics, there is a pressing need to assist researchers with enhanced environments in conducting their complex scientific experiments. 
Another application scenario for VL requiring specific support tools is remote education (e-learning).

VL can benefit from the integration of results and approaches from related areas such as tele-operation / tele-supervision federated information management, and collaborative spaces, and involves:

- Interaction between people, instruments, and information:

- Collaboration tools: Teleconferencing, chat, shared e-whiteboard, notepad, etc.;

- Scientific instruments connected to the network;

- Sharing and authorized exchange of information among collaborators;

- Large scale simulations;

- Data filtering and reduction facilities;

- Visualization tools and devices.

- Remote experiment execution and control.

- Access to information from the distributed public and proprietary sources.

- User-friendly interfaces.

- The basic necessary functionality and tools.

- Integration/fusion of distributed and diverse meta-data, data, and results.

- High-performance, secure, distributed and collaborative experimentation.

- Semi-intelligent assistance to define/perform experiments.

Tele-robotics and tele-supervision applications which make use of the Internet have been progressively increasing for a wide domain of applications (remotely operated robots and telescopes, manufacturing systems, virtual laboratories, etc.). The traditional approaches taken in this area assume a set of pre-defined services which a user can activate on simple machine and sensorial remote environments. However, if complex machinery or sensorial environments are considered to be remotely operated with maximum flexibility, a set of pre-defined services and simple monitoring/recovering procedures are not sufficient. Also, for large or variable timedelays and low availability of the communication channels, remote low level closed loop control is impractical and higher levels of control (such as supervisory control, for example) and autonomy are required. So, more sophisticated and more reliable solutions have to be found leading to an increase of the autonomy of the equipment being operated, yet preserving a high degree of flexibility. One promising approach consists of applying intelligent mobile agents [16]. Adaptable agents, carrying high level missions, can adapt themselves to the available resources in the visited places, allowing for a higher autonomy namely in the case of a temporary unavailability of network connections. Agents can also provide a facility to implement assistance mechanisms and to support asynchronous cooperation.

Recent advances in networking, high performance computing and resource management have introduced new possibilities for secure communication and computation intensive resource management [1], [9]. The GRID is a world-wide effort in this area, which takes advantage of improvements in the overall network bandwidth, and adds a new dimension to the distributed computing. Through the GRID environment, a large number of Unix-based workstations and supercomputers can be connected in an efficient way, offering users a vast amount of computational power. However, the development of communication infrastructures such as the GRID architecture and the supporting GRID middle-ware (e.g. the GLOBUS 
toolkit), are on going world-wide efforts which will not be completed any time soon. Therefore, although GRID is promising as a foundation for networked organizations, still many of its facilities are under development, and in specific at its current state, GRID falls short of supporting the necessary base for collaboration among autonomous business-oriented organizations. Unlike the pure scientific research, many organizations are keen about their autonomy and their rights to both their proprietary data and local resources, as it is also required in most applications within the VE/VO paradigm. Therefore, GRID is so far mostly being used as a partial infrastructure, on top of which, depending on the application, other functionality is developed.

Some important open research topics in VL include:

口 Integration of component technologies into a coherent platform.

a Coordinated and dynamic resource sharing for collaborative problem solving Direct access to hardware, software, data, etc.

- High-performance data integration for scientific collaboration - Identification of the best path for exchange of large data sets among VO members based on the on-line monitoring of distributed resources.

- Tele-supervision and tele-operation assisted by intelligent mobile agents.

- Facilities for dynamic definition of information (and services) access rights / different visibility levels for different VL community members - Encouraging the joint developments while preserving rights, responsibilities, and liabilities.

- Definition of adequate representation languages for Cooperation formalization - Cooperation agreement/ contracts, liability and risks negotiation, credit assignment (based on contributions), value and ownership of common developments, intangible entities (IPR, services).

- Social aspects and remote collaboration (lack of "silent language" of body motions and spatial positions).

- Asynchronous cooperation and delegation. Further developments in coordination approaches for diverse, autonomous, semi-cooperative, semireliable, heterogeneous, and evolving organizations.

- Improved mechanisms for specification of access rights and separation of virtual spaces / experiments.

\ Extended error recovery mechanisms.

- Training methodologies based on VL for manufacturing professionals.

\section{VIRTUAL COMMUNITIES SUPPORT}

When a proper cooperation nesting or breeding environment (e.g. cluster or long term network) is in place, virtual communities of practice (VCP) / virtual teams may emerge within such environment, constituting a fundamental element of value creation and sustainability. Virtual Communities and Communities of Practice are not new concepts but they acquire specific characteristics and increased importance when considered in the context of the collaborative networks of organizations. These communities, although spontaneously created, are bound to certain social rules resulting from the commitment of their members to the underlying organizations (new concept of social-bound VCPs) (Fig. 2). 


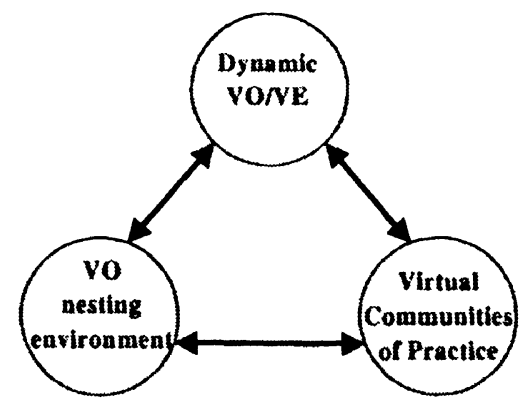

Figure 2 - VCPs in the context of VOs and cooperation nesting environments

This is the case, for instance, in concurrent or collaborative engineering where teams of engineers, possibly located in different enterprises, cooperate in a joint project such as the co-design of a new product. A large number of computer supported cooperative tools are becoming widely available for synchronous cooperation. Some examples are teleconference, and chat tools combined with application sharing mechanisms. Considering the geographical distribution, the autonomy of the VE members, the local corporate cultures, and also the individual working preferences of the team members, it is likely that most of the activities will be carried out in an asynchronous way.

In terms of coordination, several approaches to develop flexible workflow systems have been proposed. In the case of processes mainly executed by humans, rigid forms of procedural control are not adequate. People like to keep their freedom regarding the way they work. Product design, like any other creative process evolves according to a kind of "arbitrary" flow. It is therefore necessary to also support loosely constrained sets of business processes.

The trend is followed by other communities of professionals (e.g. consultants) that share the body of knowledge of their professions such as similar working cultures, problem perceptions, problem-solving techniques, professional values, and behavior.

Another special area of application, as for instance represented by the TeleCARE project [6], is the creation of virtual communities for providing care services to elderly. These communities involve organizations and people such as care providers, health care professionals, relatives of elderly, and the elderly.

Some open research topics:

口 Development and management of shared, smart spaces for geographically distributed teams of the same or different organizations, which develop complex engineering products.

- Provision of adequate visibility and access rights definition and management.

- Understand and model multi-level relationships among VC members.

- Coordination of (asynchronous) activities performed in different places by different actors; flexible coordination models.

- Frameworks for collaboration in mobile contexts.

- Tools for community management, leadership, and creation of incentives.

a Provision of notification mechanisms regarding major events in the design / planning process (e.g. conclusion of a step by one actor).

- Mechanisms to handle Intellectual Property in VCP under social contracts. 


\section{OTHER ISSUES}

Although VOs should be addressed by multi-disciplinary teams, some early developments have been tackled separately either by the ICT community or by the organizations and management community. In particular in the European context, most of the early Esprit and IST projects were technology-driven. A few recent projects put more emphasis on the management and organizational issues (with a corresponding decrease on the ICT infrastructure) but a balanced integration between the "ICT" and the "Management and Organizational" communities still seems to be missing. One exception is present at the EC-funded THINKcreative network that involves a balanced composition of experts from these two main areas.

In addition to the mentioned aspects, there are other important factors to be considered in VO, such as the social, ethical, and educational issues. The barriers for implantation of VOs are more of a socio-organizational and command logic than of technological nature. One of the early attempts to combine a "sociology" component with the technology development was represented in the Esprit PRODNET II [3].

Although there is a growing awareness for the advantages of taking a multidisciplinary approach, it is important to not under-estimate the difficulties of putting together communities with different backgrounds, different language and culture, and different approaches to problem solving. Even within the engineering area it is not always easy to reach a common language and understanding. For instance, many of the European projects on VO, although ICT-based, are mostly developed by industrial-, mechanical-, or electrical-engineers, where there is, in many cases, a "divorce" between this community and the software engineering community. The difficulties are much bigger when other non-engineering communities are put together with engineers.

There is therefore a need for an integrated multi-disciplinary approach leading to truly joint work (mutual understanding and mutual respect for the other areas!) among: Theoreticians, System engineers and ICT developers, Social \& organizational experts, Economists, Standardization bodies, and the Application Domain experts. In order to make this possible, there is also a need for further investment in formal languages - a must in order to ensure mutual understanding in a complex multi-disciplinary domain.

Furthermore, there is a need to elaborate new individual (social) protection mechanisms - agility in partnering and establishment of relationships cannot be adopted, without adequate protection mechanisms for the involved individual. Namely, mechanisms must be introduced to guarantee some long-term stability to individuals and their families in the context of highly dynamic market changes.

\section{CONCLUSIONS}

The area of virtual organizations and networked collaborative organizations in general correspond to a very dynamic domain, where new concepts, mechanisms, infrastructures and tools are emerging at a fast pace.

There is however the need for a more systematic and comprehensive understanding of the area, namely in terms of the emerging collaborative organizations that will likely play an important role in the next $5,10,15$ years, as well as in terms of developing appropriate support infrastructures and tools. In parallel with such developments it is mandatory to reach some harmonization of 
models and approaches in order to reach inter-operability and reduce the engineering efforts still required to launch an operative VO.

Such developments shall necessarily be undertaken by teams with a multidisciplinary composition, for which the difficulties of communication / understanding among different communities should not be under-estimated.

\section{ACKNOWLEDGMENTS}

The authors acknowledge partial support to this work from the European Commission (IST THINKcreative project) and IFIP (COVE / WG 5.5) and thank other partners of these projects for their valuable contribution to the discussion of some of the topics reported here.

\section{REFERENCES}

1. Afsarmanesh, H.; Kaletas, E.C.; Hertzberger, L.O. - A Reference Architecture for Scientific Virtual Laboratories, Future Generation Computer Systems, vol. 17, 2001.

2. Broos, R.; Dillenseger, B.; Guther, A.; Leith, M. - MIAMI: Mobile intelligent agents for managing the information infrastructure, www.infowin.org/ ACTS/ANALYSYS/PRODUCTS/THEMATIC/AGENTS/ch3/miami.htm, 2000.

3. Camarinha-Matos, L.M.; Afsarmanesh, H. - Infrastructures for Virtual Enterprises - Networking Industrial Enterprises, Kluwer Academic Publishers, ISBN 0-7923-8639-6, Oct 1999.

4. Camarinha-Matos, L. M.; Afsarmanesh, H. - Service Federation in Virtual Organizations, Proceedings of PROLAMAT"01, 7-10 Nov 2001, Budapest, Hungary.

5. Camarinha-Matos, L. M. and Afsarmanesh, H. - Virtual Enterprise Modeling and Support Infrastructures: Applying Multi-Agent Systems Approaches, in Multi-Agent Systems and Applications, M. Luck, V. Marik, O. Stpankova, R. Trappl (eds.), Lecture Notes in Artificial Intelligence LNAI 2086, Springer, ISBN 3-540-42312-5, July 2001.

6. Camarinha-Matos, L.M.; Afsarmanesh, H. - Design of a virtual community infrastructure for elderly care, in Collaborative Business Ecosystems and Virtual Enterprises, Kluwer Academic Publishers, May 2002.

7. Camarinha-Matos, L.M.; Afsarmanesh, H.; Rabelo, J. - Infrastructure developments for agile virtual enterprises, to appear in J. Computer Integrated Manufacturing, 2002.

8. Goranson, H.T., 1999 - The Agile Virtual Enterprise - Cases, metrics, tools. Quorum Books, ISBN 1-56720-264-0.

9. Kaletas, E. C.; Afsarmanesh, H. ; Hertzberger, L. O. - Virtual Laboratories and Virtual Organizations supporting biosciences, in Collaborative Business Ecosystems and Virtual Enterprises, Kluwer Academic Publishers, May 2002.

10. Li, Y.; Huang, B.; Liu, W.; Wu, C.; Gou, H.- Multi-agent system for partner selection of virtual enterprise, Proceedings of the World Computer Congress 2000, Track on Information Technology for Business Management, R. Gan (Ed.), Publishing House of Electronics Industry, ISBN 3-901882-057, Beijing, China, 21-25 Aug.2000.

11. NIIIP. www.niiip.org.

12. Rabelo, R.; Afsarmanesh, H.; Camarinha-Matos, L.M. - Federated multi-agent scheduling in virtual enterprises, in E-business and Virtual Enterprises, Kluwer Academic Publishers, Oct 2000.

13. Rabelo, R.; Camarinha-Matos, L.M.; Vallejos, R. - Agent-based brokerage for virtual enterprise creation in the moulds industry, in E-business and Virtual Enterprises, Kluwer Academic Publishers, ISBN 0-7923-7205-0, pp.281-290, Oct 2000.

14. Rocha, A.; Oliveira, E. - An electronic market architecture for the formation of virtual enterprises, in [3], 1999.

15. SUN - JINI Technology Architectural Overview, www.sun.com/jini/whitepapers/architecture.html, Jan 1999.

16. Vieira, W.; Camarinha-Matos, L.M. - Adaptive mobile agents: Enhanced flexibility in Internet-based remote operation, in Advances in Networked Enterprises, Kluwer Academic Publishers, ISBN 07923-7958-6, Sept 2000.

17. WfMC - Workflow Management Coalition, 1994 - The Workflow Reference Model - Document Nr. TC00 - 1003, Issue 1.1, Brussels Nov 29.

18. Zarli, A.; Poyet, P. - A framework for distributed information management in the virtual enterprise: The VEGA project, in [3], 1999. 